\title{
Online Appnedix to: The Relation between Behavior under Risk and over Time
}

\author{
Anujit Chakraborty* Yoram Halevy $^{\dagger} \quad$ Kota Saito $^{\ddagger}$
}

September 4, 2019

\section{A Diminishing Impatience does not imply Weak Certainty Effect}

The following counter-example proves that Diminishing Impatience does not imply Weak Certainty Effect (and hence by Theorem 2 also does not imply Delay Independent Diminishing Impatience). If (5.2) implied (3.4), then (5.2) would also imply that $\forall r \in(0,1)$ and $\forall m, n \in \mathbb{N}$

$$
g\left(r^{m+n}\right) \geq g\left(r^{m}\right) g\left(r^{n}\right)
$$

We will rewrite these expressions in an additive form by defining $f(x)=$ $-\log \left(g\left(e^{-x}\right)\right) \Longleftrightarrow g(x)=e^{-f(-\log x)}$. Then $f:(0, \infty) \rightarrow(0, \infty)$ is differentiable

${ }^{*}$ University of California, Davis, 1 Shields Ave, CA 95616, USA. E-mail: chakraborty@ucdavis.edu. Web: sites.google.com/site/anujit2006

${ }^{\dagger}$ Department of Economics, University of Toronto, 150 St. George Street Toronto, ON M5S 3G7 Canada. E-mail: yoram.halevy@utoronto.ca. Web: yoramhalevy.faculty.economics.utoronto.ca. Financial support from SSHRC (435-2012-0577, 4352017-0148) is gratefully acknowledged.

${ }^{\ddagger}$ California Institute of Technology, 1200 E California Blvd, MC 228-77 Pasadena, CA 91125, USA. E-mail: saito@caltech.edu. Web: people.hss.caltech.edu/ saito. Financial support from NSF (SES1558757) is gratefully acknowledged. 
and increasing, just like the function $g$. The inequalities under consideration are now:

$$
\begin{aligned}
\forall t \in \mathbb{N} \text { and } \forall r \in(0,1), g\left(r^{t+1}\right) & >g(r) g\left(r^{t}\right) \\
\Longleftrightarrow e^{-f\left(-\log \left(r^{t+1}\right)\right)} & >e^{-f\left(-\log \left(r^{t}\right)\right)} e^{-f(-\log (r))} \\
\Longleftrightarrow f(-(t+1) \log (r)) & <f(-t \log (r))+f(-\log (r))
\end{aligned}
$$

Now, defining $x:=-\log (r) \in(0, \infty)$ for $r \in(0,1)$.

$$
f((t+1) x)<f(t x)+f(x)
$$

Further, the boundary conditions $g(0)=0$ and $g(1)=1$ translate to $f(0)=0$ and $f(\infty)=\infty .^{1}$

Similarly, (A.1) converts to

$$
f(m x+n x) \leq f(m x)+f(n x) \quad \forall x \in(0, \infty) \text { and } \forall m, n \in \mathbb{N}
$$

Summing it up, (5.2) implies (A.1), if and only if (A.2) implies (A.3). The next step is to propose a function $f$ which would satisfy (A.2) on all points of its domain, but, for some $x \in \mathbb{R}$ and some $m, n \in \mathbb{N}$,

$$
f(m x+n x)>f(m x)+f(n x)
$$

Instead of providing the function $f$, we propose it's derivative $h$, so $f$ can be calculated as $f(x)=\int_{0}^{x} h(x) d x .^{2}$ Let, $k=\frac{20}{1+\sin (\pi / 2-.0001)}$ and $\delta=$ $50 k \pi \cos (\pi / 2-.0001) \approx .157$.

\footnotetext{
${ }^{1}$ Using the extended real line $(\mathbb{R} \cup \infty)$

${ }^{2}$ Recall that $f(0)=0$.
} 
Let,

$h(x)=\left\{\begin{array}{l}11+(1-x) \delta \\ 1+\frac{k}{2}+\frac{k}{2} \sin 100 \pi\left(1+\frac{\pi / 2-.0001}{100 \pi}-x\right) \\ 1 \\ 4+3 \sin 100 \pi(x-2) \\ 7 \\ 4+3 \sin 100 \pi(2.5-x) \\ 1 \\ 4+3 \sin 100 \pi(x-3) \\ 7 \\ 4+3 \sin 100 \pi(5-x) \\ 1\end{array}\right.$

For $x<1$

For $1 \leq x \leq 1.005+\frac{\pi / 2-.0001}{100 \pi}$

For $1.005+\frac{\pi / 2-.0001}{100 \pi}<x<2-.005$

For $2-.005 \leq x \leq 2+.005$

For $2+.005<x<2.5-.005$

For $2.5-.005 \leq x \leq 2.5+.005$

For $2.5+.005<x<3-.005$

For $3-.005 \leq x \leq 3+.005$

For $3+.005<x<5-.005$

For $5-.005 \leq x \leq 5+.005$

For $x>5+.005$

$f$ is increasing, twice differentiable and $f(\infty)=\infty . h(x)$ is plotted in Figure A.1.

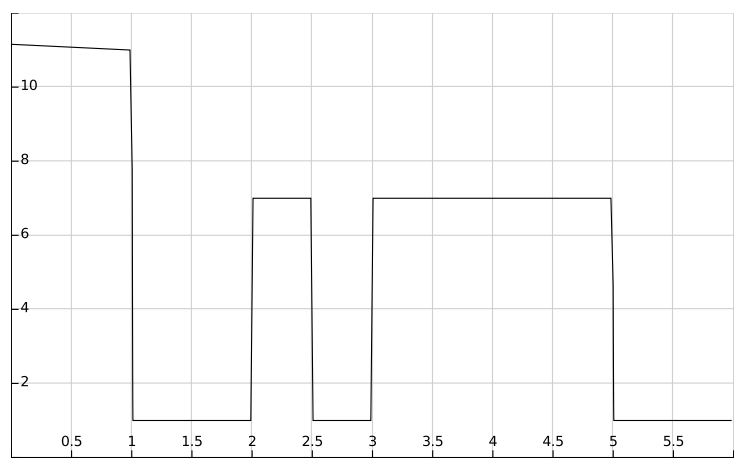

Figure A.1: The function $h$.

We next show that (A.2) holds.

Lemma 1. $\forall t \in \mathbb{N}, \forall x \in \mathbb{R}, \int_{0}^{x} h(x) d x>\int_{t x}^{(t+1) x} h(x) d x$.

Proof. The most intuitive way to check the claim would be to notice that 
the sinusoids introduced hardly perturb the area under the curve. Figure A.2 illustrates the point in a more clear fashion by considering the function $h$ for a small part of the real line. For all practical purposes, one could go about checking the inequalities by replacing the sinusoid (in black) in Figure 1 by a corresponding discontinuous function $(\bar{h}(x)=7$ for $x \leq 2.5, \bar{h}(x)=1$ for $x>2.5$ as drawn in red). The area between the two curves in $[2.495,2.5]$ is only $\left(.005 * 3-\frac{3}{100 \pi}\right) \approx .005$. Therefore, as long as the inequalities hold with a large enough margin, this intuitive method of approximating sinusoids with flat lines works fine. The area between the two curves in $[2.5,2.505]$ is also $(.005 * 3-$ $\left.\frac{3}{100 \pi}\right)$. Thus, the two approximations in $[2.495,2.505]$ are equal and opposite in direction, and the areas under the red and black curves in this region are equal. During our analysis, in some cases there will be multiple approximations in opposite directions which would partially or completely cancel each other out.

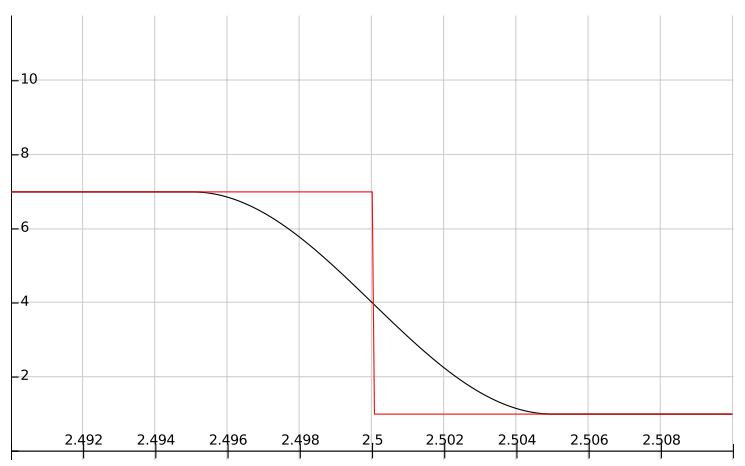

Figure A.2: Function $h$ approximated in a sinusoidal region

Utilizing this intuition more rigorously, one can create upper bounds and lower bounds on $\int_{t x}^{(t+1) x} h(x) d x$ and $\int_{0}^{x} h(x) d x$ respectively to complete the proof. For $0<x \leq 1, \int_{0}^{x} h(x) d x>\int_{t x}^{(t+1) x} h(x) d x$ is obvious, as $[0, x]$ contains the highest values obtained by $h(x)$ on the real line.

For, $1<x \leq \frac{5}{3}, \int_{0}^{x} h(x) d x=\int_{0}^{1} h(x) d x+\int_{1}^{x} h(x) d x>\frac{1}{2}(11+11+\delta)+(x-1)=$ $10+\frac{\delta}{2}+x .^{3}$ The inequality holds because $h(x) \geq 1$ with strict inequality for $1 \leq x<1.005+\frac{\pi / 2-.0001}{100 \pi}$, and hence $\int_{1}^{x} h(x) d x>x-1$. In the interval

\footnotetext{
${ }^{3} \delta=50 k \pi \cos (\pi / 2-.0001)=.157$ (approximately)
} 
$[t x,(t+1) x], h(x) \leq 7$ and after mutual canceling out there are no more than 3 sinusoidal perturbations which could increase the area under the curve. Hence, $\int_{t x}^{(t+1) x} h(x) d x<7 x+3\left(.015-\frac{3}{100 \pi}\right)=6 x+x+3\left(.015-\frac{3}{100 \pi}\right) \leq$ $6\left(\frac{5}{3}\right)+x+3\left(.015-\frac{3}{100 \pi}\right)=10+x+3\left(.015-\frac{3}{100 \pi}\right)$.

For $\frac{5}{3} \leq x \leq 2, \int_{0}^{x} h(x) d x>10+\frac{\delta}{2}+x$ as before. On the other hand, using the same line of logic as before, $\int_{x}^{2 x} g(x) d x<1 . x+6[(4-3)+(2.5-$ $2)]+3 .\left(.015-\frac{3}{100 \pi}\right)=9+x+3 .\left(.015-\frac{3}{100 \pi}\right)$. Similarly, $\int_{2 x}^{3 x} h(x) d x \leq$ $1 . x+6\left[5-2 \cdot \frac{5}{3}\right]+3 .\left(.015-\frac{3}{100 \pi}\right)=10+x+3 .\left(.015-\frac{3}{100 \pi}\right)$.

Similarly for larger values of $x$, it can be shown that $\int_{0}^{x} h(x) d x>\int_{t x}^{(t+1) x} h(x) d x$. (follows trivially for $x \geq 5$.)

Now complete the counter-example:

$$
\int_{0}^{2} h(x) d x<12+\frac{\delta}{2}+\left\{.01 * 10+\left(.015-\frac{3}{100 \pi}\right)\right\}<14-2\left(.015-\frac{3}{100 \pi}\right)=\int_{3}^{5} h(x) d x
$$

The first inequality follows from setting an upper bound on the sinusoidal perturbation introduced around $1 .{ }^{4}$ Therefore, $f(5)>f(2)+f(3)$, which provides us with the counter-example to equation (A.3) and hence, to equation (A.1). In other words, as (A.2) does not imply (A.3), (5.2) does not imply (A.1), and hence, (5.2) does not imply (3.4).

That is, even if for all $t \in \mathbb{N}$ and for all $r \in(0,1): g\left((1-r)^{t+1}\right)>g((1-$ $\left.r)^{t}\right) g((1-r))$ it does not imply that $\forall p, q \in(0,1): g(p q) \geqslant g(p) g(q)$.

\footnotetext{
${ }^{4}$ This particular sinusoid dies down after $1.005+\frac{\pi / 2-.0001}{100 \pi}<1.01$ and never rises above the $h(x)=1$ line by more than 6 units.
} 


\section{B Extension: temporal rewards with pure time discounting}

In the paper, we have defined time preferences $\left\{\succsim_{d}\right\}$ as follows:

$$
[x, t] \succ_{d}[y, s] \Leftrightarrow(x, p(t \mid d)) \succ^{r}(y, p(s \mid d)) .
$$

This definition assumes that the agent discounts future payoffs only because the future payoffs are uncertain due to the hazard rate. In this section, we show that a result similar to Theorem 1 holds even when we allow the agent to discount future payoffs not only because the future payoffs are uncertain but also because of "pure" time discounting (i.e., the agent intrinsically prefers early rewards to later rewards).

We would need the following new notations to separate out the two effects of time, one of pure discounting and the other of temporal hazard:

- $(x, \tau)$ : sure payoff of $x \in X$ after waiting $\tau \in T$ periods.

- $((x, \tau), p)$ : the lottery which gives $(x, \tau) \in X \times T$ with probability $p \in$ $[0,1]$ and gives nothing with the probability $1-p$. (Temporal Lotteries)

The reader should interpret the former as a counterfactual choice-object, where by choosing $(x, \tau)$, the agent can consume $x$ for sure but the reward arrives $\tau$ periods later, and hence is subjected to pure discounting. The latter is a temporal lottery that provides $(x, \tau)$ with probability $p$. Finally, we assume that the agent at decision period $d$ discounts a future reward $[x, t] \in X(d)$ not only because the future reward is uncertain (i.e., the probability of consuming $x$ is $p(t \mid d)$ ) but also because the agent needs to wait $t-d$ periods until $x$ is obtained. Therefore the agent's time preferences can be defined as follows:

Assumption 1: For all $d \in T$ and $[x, t],[y, s] \in X(d)$

$$
[x, t] \succsim_{d}[y, s] \Leftrightarrow((x, t-d), p(t \mid d)) \succsim^{r}((y, s-d), p(s \mid d)) .
$$

We are going to additionally assume: 
Assumption 2: For any $x, y \in X, \tau, \gamma \in T$, and $p, q \in[0,1]$

$$
((x, \tau), p) \succsim((y, \gamma), q) \Leftrightarrow((x, \tau+\sigma), p) \succsim((y, \gamma+\sigma), q)
$$

This assumption means that equally postponing two temporal lotteries (i.e., $\sigma$ periods) does not reverse the preference between them. This axiom is slightly stronger than an axiom (Axiom B2) of Fishburn and Rubinstein (1982), in which they assume $\tau=\gamma$.

Common-ratio effect, defined as a property of risk preferences $\left\{\succsim^{r}\right\}$ on atemporal lotteries in the main paper, is now extended to the domain of temporal lotteries.

Definition 1. $\succsim^{r}$ is said to exhibit Strict Common-Ratio Effect, if for any $x, y \in X$ and $p, q \in(0,1]$ such that $x<y, p<q$, and $((x, \tau), p) \sim^{r}((y, s), q)$

(a) $((x, \tau), p r) \succ^{r}((y, s), q r)$ for all $r<1$.

(b) $((x, \tau), p r) \prec^{r}((y, s), q r)$ for all $r>1$ and $q r<1$

Strict Certainty Effect can be defined as Strict Common-Ratio Effect with $q=1$. Similarly, Independence can be defined as follows:

$$
((x, \tau), p) \succ^{r}((y, s), q) \Longrightarrow((x, \tau), p r) \succ^{r}((y, s), q r)
$$

The definitions of temporal reversals, present-biased temporal reversal, and temporally unbiased are the same as in the main paper.

Theorem 1. Under Assumptions 1 and 2,

(i) $\succsim^{r}$ exhibits Strict Common Ratio Effect (a) and (b) iff $\left\{\succsim_{d}\right\}_{d \in T}$ exhibit Temporal Reversal (a) and (b), respectively.

(ii) $\succsim^{r}$ exhibits Strict Certainty Effect (a) and (b) iff $\left\{\succsim_{d}\right\}_{d \in T}$ exhibit Present Biased Temporal Reversal (a) and (b), respectively.

(iii) $\succsim^{r}$ satisfies the Independence Axiom iff $\left\{\succsim_{d}\right\}_{d \in T}$ is Temporally Unbiased. 
Proof. First we show (i). Step 1: First showing that Strict Common Ratio Effect (a) implies Temporal Reversal (a). Suppose that $d^{\prime}<d<t<s$.

$$
\begin{aligned}
{[x, t] \sim_{d}[y, s] } & \Longleftrightarrow((x, t-d), p(t \mid d)) \sim_{r}((y, s-d), p(s \mid d)) \\
& \left.\Longrightarrow\left((x, t-d), p(t \mid d) p\left(d \mid d^{\prime}\right)\right) \prec_{r}\left((y, s-d), p(s \mid d) p\left(d \mid d^{\prime}\right)\right)\right) \\
& \left.\Longrightarrow\left((x, t-d), p\left(t \mid d^{\prime}\right)\right) \prec_{r}\left((y, s-d), p\left(s \mid d^{\prime}\right)\right)\right) \\
& \left.\Longrightarrow\left(\left(x, t-d^{\prime}\right), p\left(t \mid d^{\prime}\right)\right) \prec_{r}\left(\left(y, s-d^{\prime}\right), p\left(s \mid d^{\prime}\right)\right)\right) \\
& \Longrightarrow \quad x, t] \prec_{d}[y, s] .
\end{aligned}
$$

The second implication follows from $p\left(d \mid d^{\prime}\right)<1$ and Strict Common-Ratio Effect (a), and the second last implication follows from Assumption 2.

Step 2: Now showing that Temporal Reversal (a) implies Common-Ratio Effect (a): Fix $p, q, r$ such that $p<q$ and $r<1$. Choose $t, s, d, d^{\prime}$ such that $d^{\prime}<d<t<s$ and $p(t \mid d)=p, p(s \mid d)=q$, and $p\left(d \mid d^{\prime}\right)=r<1$. Then $p\left(t \mid d^{\prime}\right)=p r$ and $p\left(s \mid d^{\prime}\right)=q r$. Moreover,

$$
\begin{aligned}
((x, t), p) \sim_{r}((y, s), q) & \Longleftrightarrow \quad((x, t), p(t \mid d)) \sim_{r}((y, s), p(s \mid d)) \\
& \Longleftrightarrow[x, t] \sim_{d}[y, s] \\
& \Longleftrightarrow \quad[x, t] \prec_{d^{\prime}}[y, s] \\
& \Longrightarrow\left(\left(x, t-d^{\prime}\right), p\left(t \mid d^{\prime}\right)\right) \prec_{r}\left(\left(y, s-d^{\prime}\right), p\left(s \mid d^{\prime}\right)\right) \\
& \Longrightarrow\left(\left(x, t-d^{\prime}\right), p r\right) \prec_{r}\left(\left(y, s-d^{\prime}\right), q r\right) \\
& \Longrightarrow((x, t), p r) \prec_{r}((y, s), q r) .
\end{aligned}
$$

Steps 1 and 2 show that Strict Common-Ratio Effect (a) is equivalent to Temporal Reversal (a). In the same way, we can show that Strict Common Ratio Effect (b) is equivalent to Temporal Reversal (b). Hence, statement (i) holds. In the same way, assuming $q=1$ and $d=t$, we obtain statement (ii). Finally, we show statement (iii).

Step 3: Independence implies Temporally Unbiased. Suppose that $d^{\prime}<$ 
$d<t<s$

$$
\begin{aligned}
{[x, t] \succsim_{d}[y, s] } & \Longleftrightarrow((x, t-d), p(t \mid d)) \succsim_{r}((y, s-d), p(s \mid d)) \\
& \left.\Longleftrightarrow\left((x, t-d), p(t \mid d) p\left(d \mid d^{\prime}\right)\right) \succsim_{r}\left((y, s-d), p(s \mid d) p\left(d \mid d^{\prime}\right)\right)\right) \\
& \left.\Longleftrightarrow\left((x, t-d), p\left(t \mid d^{\prime}\right)\right) \succsim_{r}\left((y, s-d), p\left(s \mid d^{\prime}\right)\right)\right) \\
& \left.\Longleftrightarrow\left(\left(x, t-d^{\prime}\right), p\left(t \mid d^{\prime}\right)\right) \succsim_{r}\left(\left(y, s-d^{\prime}\right), p\left(s \mid d^{\prime}\right)\right)\right) \\
& \Longleftrightarrow[x, t] \succsim_{d}[y, s] .
\end{aligned}
$$

The second equivalence holds by Independence. The second to the last equivalence holds by Assumption 2.

Step 4: Temporally Unbiased implies Independence. Fix $p, q, r$ such that $p<q$ and $r<1$. Choose $t, s, d, d^{\prime}$ such that $d^{\prime}<d<t<s$ and $p(t \mid d)=p$, $p(s \mid d)=q$, and $p\left(d \mid d^{\prime}\right)=r<1$. Then $p\left(t \mid d^{\prime}\right)=p r$ and $p\left(s \mid d^{\prime}\right)=q r$, Moreover,

$$
\begin{aligned}
((x, t), p) \succsim_{r}((y, s), q) & \Longleftrightarrow((x, t), p(t \mid d)) \succsim_{r}((y, s), p(s \mid d)) \\
& \Longleftrightarrow[x, t] \succsim_{d}[y, s] \\
& \Longleftrightarrow[x, t] \succsim_{d^{\prime}}[y, s] \\
& \Longleftrightarrow\left(\left(x, t-d^{\prime}\right), p\left(t \mid d^{\prime}\right)\right) \succsim_{r}\left(\left(y, s-d^{\prime}\right), p\left(s \mid d^{\prime}\right)\right) \\
& \Longleftrightarrow((x, t), p r) \succsim_{r}((y, s), q r) .
\end{aligned}
$$

The third equivalence holds by Temporally Unbiased and the last equivalence holds by Assumption 2. 


\section{$\mathrm{C}$ Extension: non-constant hazard rate}

There are two types of intertemporal preference reversals. Violations of Stationarity (static reversals) is a choice pattern at a fixed decision time, where the relative impatience between two periods increases as they are equallyshifted closer to the decision time. Violations of Time-consistency (dynamic reversals), on the other hand, fixes the two periods between which choice is to be made, but as the decision time shifts forward closer to the the two periods, the relative impatience revealed by choices increases. When temporal preferences are Time-invariant (Halevy, 2015) (i.e, when preferences depend only on the temporal distance between decision and consumption time), dynamic and static reversals coincide.

In our treatment of preferences over temporal rewards, we consider dynamic reversals as preferences $\left\{\succsim_{d}\right\}_{d \in T}$ depend on the decision time $d$. On the other hand, in our treatment of preferences over consumption streams, we consider static reversals since the discounting function $D$ is defined over the temporal distance but not on the decision date, following the standard formulation of the literature. In the main paper, we assume that the hazard rate is constant. This implies that preferences are Time-invariant and therefore each formulation captures dynamic as well as static reversals.

If we allow for an arbitrary hazard rate (i.e., $r_{t} \neq r_{s}$ for calendar times $t \neq s$ ), static and dynamic reversals would no longer coincide. However, in the temporal-rewards domain, Theorem 1 holds exactly in the same way as long as the function $p(\cdot)$ satisfies the following standard conditions: (i) $p(0)=1$; (ii) $p(+\infty)=0$; (iii) $p$ is continuous and strictly decreasing. ${ }^{5}$ In the following, we discuss how our results for consumption streams could be extended to nonconstant hazard rate, in the context of dynamic reversals.

Consider the dynamic problem where the DM knows that conditional on reaching some period $\tau \geq 0$, the consumption in the next period $\tau+1$ will be available with probability of $1-r_{\tau}$ (so the hazard rate between periods $\tau$ and $\tau+1$ is $\left.r_{\tau} \in[0,1]\right)$. The equivalent of Diminishing Impatience in the dynamic

\footnotetext{
${ }^{5}$ Similar findings are discussed in Halevy (2004; 2015).
} 
context is $\frac{D_{t^{\prime}}(t)}{D_{t^{\prime}}(t+1)}<\frac{D_{t}(t)}{D_{t}(t+1)}$ for all $t, t^{\prime} \in \mathbb{N}$ and $t^{\prime}<t$, where

$$
D_{s}(t)=\delta^{t-s} g\left(\frac{p(t)}{p(s)}\right)=\delta^{t-s} g\left(\Pi_{\tau=s}^{t-1}\left(1-r_{\tau}\right)\right)
$$

is the composite discounting of some period $t \geq s$ when the decision time is $s$, and $p(\tau)$ is the prior probability of reaching at least period $\tau$. We adopt the convention that $\Pi_{\tau=t}^{t-1}\left(1-r_{\tau}\right)=1$.

Note that this actually has to be interpreted slightly differently from our previous (static) Diminishing Impatience property. This new property states that the DM's impatience between consumption at any two consecutive periods $t$ and $t+1$ is highest when the decision period is $t$ (i.e., considering immediate consumption versus consumption delayed by one period). We call this property Dynamic Diminishing Impatience.

Similarly, define Dynamic Strongly Diminishing Impatience as $\frac{D_{t^{\prime \prime}}(t)}{D_{t^{\prime \prime}}(t+1)}<$ $\frac{D_{t^{\prime}}(t)}{D_{t^{\prime}}(t+1)}$ for all $t, t^{\prime \prime}, t^{\prime} \in \mathbb{N}$ and $t^{\prime \prime}<t^{\prime} \leq t$.

The following implications follow directly from the definitions:

(i) Dynamic Diminishing Impatience holds if and only if for every $r_{\tau} \in(0,1)$ and $t \in \mathbb{N}$ :

$$
g\left(\Pi_{\tau=t^{\prime}}^{t}\left(1-r_{\tau}\right)\right)>g\left(\Pi_{\tau=t^{\prime}}^{t-1}\left(1-r_{\tau}\right)\right) g\left(\left(1-r_{t}\right)\right)
$$

(ii) Dynamic Strongly Diminishing Impatience holds if and only if for every $\left\{\left\{r_{\tau}\right\}_{\tau \in \mathbb{N}}: r_{\tau} \in(0,1)\right\}$ and $t, t^{\prime \prime}, t^{\prime} \in \mathbb{N}$ and $t^{\prime \prime}<t^{\prime} \leq t:$

$$
\frac{g\left(\Pi_{\tau=t^{\prime \prime}}^{t}\left(1-r_{\tau}\right)\right)}{g\left(\Pi_{\tau=t^{\prime \prime}}^{t-1}\left(1-r_{\tau}\right)\right)}>\frac{g\left(\Pi_{\tau=t^{\prime}}^{t}\left(1-r_{\tau}\right)\right)}{g\left(\Pi_{\tau=t^{\prime}}^{t-1}\left(1-r_{\tau}\right)\right)}
$$

Theorem 2. Consider a DM whose preferences are represented by (2.1) with $g(\cdot)$. The following hold :

(i) Dynamic Strongly Diminishing Impatience implies Strict Common-Ratio Effect.

\footnotetext{
${ }^{6}$ Continuity of $g()$ is no longer needed
} 
(ii) Dynamic Diminishing Impatience implies Strict Certainty Effect.

(iii) The converses of 1 and 2 are easy to show and are omitted.

Proof. We first show claim (i). We will show that for any $p, q \in(0,1)$ and $\ell \in(0,1],(3.3)$ in Remark 1 holds. Let $q<p$ without loss of generality. Take $t^{\prime \prime}=0, t^{\prime}=1, t=2$, and $r_{0}=1-p, r_{1}=1-\ell, r_{2}=1-q$. Dynamic Strongly Diminishing Impatience implies that

$$
\begin{aligned}
& \frac{g\left(\left(1-r_{0}\right)\left(1-r_{1}\right)\left(1-r_{2}\right)\right)}{g\left(\left(1-r_{0}\right)\left(1-r_{1}\right)\right)}>\frac{g\left(\left(1-r_{1}\right)\left(1-r_{2}\right)\right)}{g\left(1-r_{1}\right)} \\
& \Longleftrightarrow \frac{g(p q \ell)}{g(p \ell)}>\frac{g(q \ell)}{g(\ell)} \\
& \Longleftrightarrow \frac{g(\ell)}{g(p \ell)}>\frac{g(q \ell)}{g(p q \ell)}
\end{aligned}
$$

Part (2) is a special case of (1), where $\ell=1$ (i.e., $r_{1}=0$ ), and Dynamic Diminishing Impatience is used instead of Dynamic Strongly Diminishing Impatience.

\section{References}

Fishburn, Peter C. and Ariel Rubinstein, "Time Preference," International Economic Review, October 1982, 23 (3), 677-694.

Halevy, Yoram, "Diminishing impatience: disentangling time preference from uncertain lifetime," 2004.

_ , "Time Consistency: Stationarity and Time Invariance," Econometrica, January 2015, 83 (1), 335-352. 